\title{
CUSTOS DA OBTENÇÃO DA BOLSA DE PLAQUETAS POR AFÉRESE EM UM HEMOCENTRO DO INTERIOR DE SÃO PAULO
}

\section{COSTS OF OBTAINING THE BAG OF PLATELETS BY APHERESIS IN A BLOOD CENTER OF THE INNER SÃO PAULO}

\section{COSTOS DE OBTENER UNA BOLSA DE PLAQUETAS DE AFÉRESIS EN UN CENTRO DE SANGRE EN EL INTERIOR DE SÃO PAULO}

\author{
Duelene Ludimila Nogueira1', Meline Rossetto Kron Rodrigues², Claudia Cristina de Oliveira Alcarde ${ }^{3}$, \\ Laura Tiaki Fujihara ${ }^{3}$, Alba Cristina Albano ${ }^{3}$, Gislene Cristina Mastranjo de Oliveira ${ }^{3}$, Fernando Aparecido Oliveira ${ }^{3}$, \\ Tamires Correa de Paula1, Silvana Andréa Molina Lima ${ }^{4}$
}

\begin{abstract}
RESUMO
Objetivo: Identificar e calcular os custos diretos para a geração de uma bolsa de plaquetas por aférese em um Hemocentro do interior de São Paulo. Método: Trata-se de um estudo observacional, retrospectivo e de abordagem quantitativa com análise descritiva dos dados realizado no Hemocentro da Unesp-Botucatu. Para obtenção dos custos diretos foram incluídas todas as bolsas coletadas por plaquetaférese no período de janeiro a dezembro de 2012, desde o momento da captação do doador até o final da coleta do hemocomponente. Resultados: Foram coletadas 289 bolsas no período estudado e os custos diretos para obtenção da bolsa por plaquetaférese foi de USD172,62. Conclusão: Concluiu-se que a análise dos custos é uma importante ferramenta de gestão e demonstrou-se a necessidade da realização de outros trabalhos na área do gerenciamento de custos devido à dificuldade de encontrar artigos e materiais publicados relacionados a custos na geração de hemocomponentes, principalmente no setor de aférese.
\end{abstract}

DESCRITORES: Serviço de Hemoterapia; Transfusão de Sangue; Remoção de Componentes Sanguíneos; Plaquetas; Custos Diretos de Serviços

\begin{abstract}
Objective: To identify and calculate the direct costs for the generation of a platelet bag by apheresis in a Blood Center in the interior of São Paulo. Method: It is an observational, retrospective and quantitative approach with a descriptive analysis of data performed at the Unesp-Botucatu Blood Center. In order to obtain the direct costs, all the bags collected by plateletpheresis were included in the period from January to December 2012, from the time of donor capture until the end of the collection of the blood component. Results: 289 bags were collected in the study period and the direct costs to obtain the bag by plateletpheresis were USD172.62. Conclusion: It was concluded that cost analysis is an important management tool and demonstrated the need to carry out other work in the area of cost management due to the difficulty of finding published articles and materials related to costs in the generation of blood components, mainly in the apheresis sector.
\end{abstract}

KEYWORDS: Hematology Service; Blood Transfusion; Blood Component Removal; Platelets; Direct Service Costs

\footnotetext{
1 Doutoranda do Programa de Pós-Graduação em Enfermagem da Faculdade de Medicina de Botucatu, UNESP-Universidade Estadual Paulista - Botucatu, SP, Brasil

2 Pós-Doutoranda do Programa de Pós-Graduação em Enfermagem da Faculdade de Medicina de Botucatu, UNESP-Universidade Estadual Paulista - Botucatu, SP, Brasil

Hemocentro de Botucatu, UNESP-Universidade Estadual Paulista - Botucatu, SP, Brasil

4 Docente Titular do Departamento de Enfermagem da Faculdade de Medicina de Botucatu, UNESP-Universidade Estadual Paulista Botucatu, SP, Brasil
} 


\section{RESUMEN}

Objetivo: identificar y calcular los costos directos para la generación de una bolsa de plaquetas de aféresis en un centro de sangre en el interior de São Paulo. Método: Este es un estudio observacional, retrospectivo y cuantitativo con análisis descriptivo de los datos realizados en el Centro de Sangre Unesp-Botucatu. Para obtener costos directos, se incluyeron todas las bolsas recolectadas por plaquetasféresis de enero a diciembre de 2012, desde el momento de la captación de los donantes hasta el final de la recolección de componentes sanguíneos. Resultados: Se recogieron un total de 289 bolsas durante el período de estudio y los costos directos para obtener la bolsa por plaquetas es de USD172.62. Conclusión: Se concluyó que el análisis de costos es una herramienta de gestión importante y se demostró la necesidad de trabajar más en el área de gestión de costos debido a la dificultad de encontrar artículos y materiales publicados relacionados con los costos en la generación de componentes sanguíneos, principalmente en el sector de aféresis.

DESCRIPTORES: Servicio de Hemoterapia; Transfusión Sanguínea; Eliminación de Componentes Sanguíneos; Plaquetas; Costos Directos de Servicios

\section{INTRODUÇÃO}

Nos últimos anos, a prática transfusional era tangida pelos riscos em adquirir-se doenças transmissíveis e os efeitos lesivos originados ao receptor, além de um alto custo associado às transfusões. Contudo, com o avanço da área clínica da Medicina Transfusional, a hemoterapia brasileira vem adotando novas tecnologias para garantir maior segurança e qualidade do ciclo sanguíneo. ${ }^{1,2}$

A prática transfusional é um processo que mesmo realizado dentro dos protocolos e perfeitamente executado, absorve riscos para seus utentes, conferindo benefícios que caminham simultaneamente com complicações designadas imediatas e tardias, oriundas de diversos fatores. ${ }^{2}$

Durante muitos anos, o sangue era coletado e não havia oportunidade de separar todos os hemocomponentes, apenas o plasma era fracionado. Com o avanço da medicina transfusional, os hemocomponentes enfim tornaram-se plausíveis de fracionamento. Essa evolução aconteceu concomitantemente a descobertas de novas doenças hematológicas, que eram diagnosticadas como deficiência em apenas um único componente sanguíneo. ${ }^{3}$

O fracionamento do sangue total traz imensos benefícios como ampliação do tempo de validade de todos os componentes sanguíneos, modo de utilização otimizado em relação ao aproveitamento e eficácia e também, diminui consideravelmente o risco de ocorrência de alguma reação transfusional imediata ou tardia. Contudo, é importante ressaltar que tanto a prescrição adequada quanto a indicação clínica são de extrema ne- cessidade. ${ }^{3}$ Os hemocomponentes são obtidos pela doação de sangue total, podendo ser por centrifugação das bolsas de sangue, ou concentrados obtidos por aférese, sendo as plaquetas o mais rotineiro. ${ }^{3}$

De ascendência grega, a aférese surgiu durante os anos 60 , e significa separar, retirar seletivamente um único componente. A aférese pode ser realizada para fins transfusionais (doação) ou terapêutico ${ }^{4}$

A plaquetaférese para fins transfusionais é o procedimento principal, onde se obtém o hemocomponente a partir de um doador único que será transfundido em um paciente, consistindo na remoção apenas das plaquetas, onde uma centrífuga de fluxo intermitente bombeia sangue para um filtro que irá filtrar somente as plaquetas e devolverá os outros hemocomponentes para o doador, por meio de ciclos cíclicos. ${ }^{4-6}$

Já a plasmaférese na modalidade terapêutica é indicada quando a substância causadora da doença está presente no plasma e pode ser removida pela troca plasmática. Sua eficácia associa-se a quantidade de plasma trocado em cada procedimento, bem como da distribuição dessa substância entre os compartimentos intra e extravascular e da velocidade de equilíbrio entre esses compartimentos. É recomendado que haja intervalo entre as sessões, pois associa-se a patologia do paciente, à velocidade de instalação da doença, da gravidade, da substância alvo a ser removida, assim como da perpetuação do estímulo à produção dessa substância.4-6

A avaliação dos custos torna-se um poderoso indicativo para a gestão, norteando decisões e planejamento de ações a serem realizadas dentro de uma instituição. $A$ operacionalização com menor custo e com bons resulta- 
dos finais, sem afetar a qualidade do produto, deve ser um objetivo institucional. ${ }^{7}$

Custo é definido como a somatória do gasto com pessoal, material, estrutura física e equipamentos, devendo ser compreendido como um poderoso instrumento gerencial e servindo para análise de desempenho, produtividade e qualidade dos serviços. ${ }^{8}$

Os custos podem ser classificados em diretos, indiretos, variáveis e fixos. Os custos diretos são gastos utilizados diretamente na produção de um produto ou execução de um procedimento, como o gasto com recurso humano para administração de uma medicação. Já o custo indireto é calculado por meio de rateio como luz, aluguel e água. Os custos fixos são custos operacionais constantes vinculados à infraestrutura da instituição como aluguel e salários. Os custos variáveis estão relacionados ao volume de produção e procedimentos como medicamentos e materiais. ${ }^{8,9}$

O desconhecimento dos custos, incluindo a meIhor combinação de recursos e a sua efetividade, dificultam as organizações a ajustarem suas atividades dentro de seus orçamentos. ${ }^{8,9}$. Os gerentes das instituições de saúde se deparam com o desafio de obter a utilização racional e eficiente dos recursos que possuem.

Sendo a doação de plaquetas por aférese um procedimento específico, pouco conhecido pela população e até mesmo por profissionais de saúde e a finitide dos recursos financeiros disponíveis na saúde, associado à escassez de dados na literatura sobre a avaliação de custos na área de Hemoterapia justifica-se a elaboração deste estudo que objetivou identificar e calcular os custos diretos para a geração de uma bolsa de plaquetas por aférese de um Hemocentro do interior do estado de São Paulo.

\section{MÉTODO}

Trata-se de um estudo observacional, retrospectivo e de abordagem quantitativa com análise descritiva dos dados, realizado no Hemocentro do Hospital das Clinicas da Faculdade de Medicina de Botucatu (HCFMB). Como critério de inclusão, definiu-se como toda bolsa de plaqueta de aférese que gerada na unidade estudada no período de janeiro a dezembro de 2012.

Para contabilização dos custos diretos com a geração de bolsas de plaquetas por aférese, foram identificados todos os gastos com exames laboratoriais, material de consumo, material médico-hospitalar e despesas com recursos humanos, gerados no período de janeiro a dezembro de 2012, desde o momento da captação do doador, até o final da coleta do hemocomponente. Os valores monetários foram obtidos a partir do Núcleo de Custos da FMB - UNESP e almoxarifado do Hemocentro.

Os recursos humanos foram quantificados proporcionalmente ao tempo de serviço em que o funcionário desempenha atividades diretamente com aférese, sendo que dentro do hemocentro estudado também são produzidos outros tipos de hemocomponentes.

Para coleta dos dados, foi elaborado um fluxograma correspondente à rotina da unidade estudada, sequenciando os procedimentos necessários segundo protocolo instituido pela unidade para coleta da bolsa de plaqueta por aférese, desde o momento da captação do doador até o término da coleta. Após a elaboração do fluxograma, houve a capitalização monetária dos custos gerados por partes, codificados e distribuídos em planilhas do Excel para melhor visualização e análise descritiva, sendo designado o preço unitário por bolsa. Os custos foram obtidos em reais ( $R \$)$ e convertidos em dólar americano (USD) com câmbio de R\$ 4,00.

Foram preservados os aspectos éticos previstos na Resolução N 466/2012 do Conselho Nacional de Saúde. O projeto foi aprovado pelo Comitê de Ética em Pesquisa da Faculdade de Medicina de Botucatu Unesp sob o protocolo 4412/2012.

\section{RESULTADOS}

No ano de 2012, no período de 12 meses, houve coleta de 289 bolsas de plaquetas por aférese. Para obtenção do custo direto referente as bolsas de aféreses geradas, elaborou-se um fluxograma para delinear o fluxo que o doador percorre para realizar a doação de plaquetas por aférese (figura 1): 


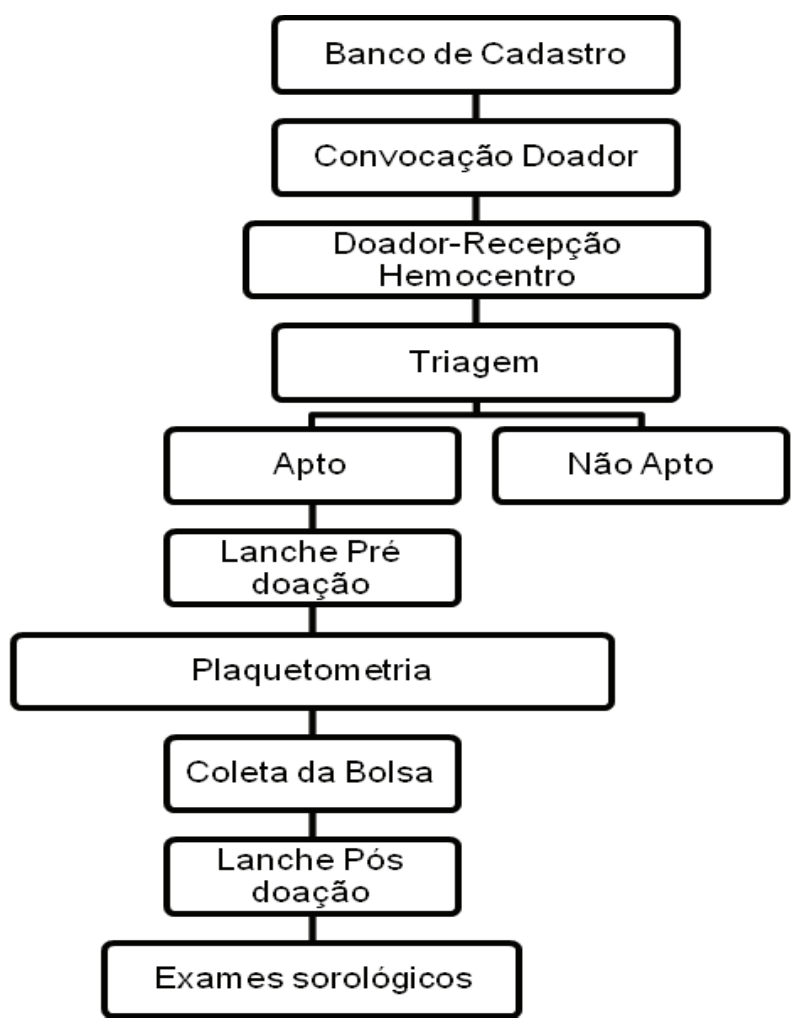

Figura 1- Fluxograma elaborado para delinear o fluxo que o doador percorre para realizar a doação de plaquetas por aférese.

Após identificar o ciclo percorrido pelo doador dentro do hemocentro, foi realizado o levantamento das atividades desempenhadas pelos servidores e quantificado os recursos consumidos para concluir a produção de uma bolsa de plaquetas. Os custos diretos foram classificados em mão-de-obra, material de consumo, material médico-hospitalar, exames e lanche.

Os valores com mão de obra foram obtidos dividindo-se a remuneração mensal do funcionário pelo tempo que ele realiza cada atividade, pois alguns funcionários do hemocentro desempenham outras atividades além da geração de bolsas de plaquetas por aférese. $O$ material de consumo, material médico-hospitalar, exame e os lanches foram obtidos quantificando-se os materiais utilizados por procedimento.

Deste modo, o custo direto para geração de uma bolsa de plaquetas por aférese, perfaz $R \$ 690,53$, conforme expresso na tabela 1.
Quadro 1- Custos diretos da geração de bolsas de plaquetas por aférese, no Hemocentro - HCFMB - UNESP, 2012.

\begin{tabular}{|l|c|}
\hline \multicolumn{1}{|c|}{ Custos diretos: } & $\begin{array}{c}\text { Valores } \\
\text { (USD)* }\end{array}$ \\
\hline $\begin{array}{l}\text { Mão de Obra (Equipe de Enfermagem, auxiliar } \\
\text { de cozinha e oficial administrativo) }\end{array}$ & 14,41 \\
\hline $\begin{array}{l}\text { Material de Consumo (Informática, } \\
\text { Escritório,Ligação e peças de reposição) }\end{array}$ & 0,84 \\
\hline Material Médico-Hospitalar & 138,94 \\
\hline Exames (laboratoriais) & 17,49 \\
\hline Lanche (pré e pós doação) & 0,94 \\
\hline Total & 172,62 \\
\hline
\end{tabular}

*Valores convertidos de reais $(\mathrm{R} \$)$ para dólar americano (USD) com câmbio de $\mathrm{R} \$ 4,00$

\section{DISCUSSÃO}

Contatou-se que o custo da bolsa de plaquetas por aférese foi de USD 172.62 ao passo que o repasse do Sistema Único de Saúde (SUS) segundo a tabela SIGTAP - Sistema de Gerenciamento da Tabela de Procedimentos, Medicamentos e OPM do SUS é de USD 45.00 por procedimento. $\mathrm{O}$ desenvolvimento tecnológico no setor da saúde tem sido responsável pelo aumento dos custos na assistência, seja pela introdução de novas técnicas, ou pela modernização das já existentes no mercado.

Em estudo realizado no mesmo serviço, com o objetivo de conhecer o perfil sociodemográfico dos doadores de plaquetas por aférese, foi identificado que no ano de 2011 houve coleta de 254 bolsas, ao passo que em 2012 foram coletadas 289 bolsas. Tal fato evidencia que foram produzidas 35 bolsas a mais do que no ano de 2011. Taxa pouco significativa, porém, já evidencia o aumento da demanda anual. ${ }^{10}$

Outro estudo realizado no hemocentro de Ribeirão Preto, que apurou custo médio do Módulo de Coleta de sangue total, constatou que o custo obtido para desempenho das atividades que compõem o Módulo 
de Coleta foi $68,75 \%$ superior ao valor pago na tabela SUS ${ }^{(11)}$. Monitorar e simplificar custos constituem pré-requisitos para a boa administração das instituições. A busca pelo menor custo, sem abalar a funcionalidade e a qualidade dos produtos ou serviços, deve ser uma meta institucional contínua. ${ }^{11-12}$

Realizar procedimentos complexos exige mão de obra qualificada e produtos importados, responsáveis por grande parte dos gastos do procedimento. ${ }^{12-14}$ No hemocentro estudado, o kit comprado para realização da aférese custa USD 138.94 dólares o que exige então a utilização racional e bem realizada desse material.

$\mathrm{Na}$ hemoterapia, a segurança transfusional está diretamente ligada à qualidade dos hemocomponentes gerados e para atingir qualidade e reduzir gastos é necessário trabalhar com uma equipe treinada e que realize os procedimentos com o mínimo de perdas possíveis. ${ }^{13-14}$

Conhecer os custos aplicando metodologias semelhantes para obtenção do custo dos outros procedimentos hemoterápicos, visando apuração do custo de uma unidade de sangue total e/ou uma unidade de he- mocomponente e transfusão, certamente fornecerá elementos para as decisões gerenciais dos serviços.

\section{CONCLUSÃO}

A hemoterapia constitui um ramo da saúde complexo e de alto nível, que necessita de recursos específicos, mão de obra qualificada e avaliação constante das atividades desempenhadas para manter a qualidade esperada. Nota-se que o repasse financeiro da entidade financiadora pode ficar abaixo do esperado para gerir os custos do hemocentro, não sendo suficiente para pagamento das despesas na geração de bolsas de plaquetas por aférese.

A dificuldade de encontrar artigos e materiais publicados relacionados a custos na geração de hemocomponentes em especial da plasmaférese, mostra a importância em se desenvolver estudos como esse, afim de pautar e embasar a busca pela melhora dos recursos dentro da hemoterapia e fundamentar estratégias para aumentar o valor pago pela tabela SUS. 


\section{REFERÊNCIAS:}

1. De Mattia D, De Andrade SR. Cuidados de enfermagem na transfusão de sangue: um instrumento para monitorização do paciente. Texto \& Contexto Enfermagem, 2016; 25.2. DOI: http://dx.doi. org/10.1590/0104-07072016002600015.

2. Forster F. et al. Percepção dos enfermeiros quanto à assistência de enfermagem no processo transfusional. Enfermagem em foco. 2018;9(3). DOI: https:// doi.org/10.21675/2357-707X.2018.v9.n3.1509

3. Reis VN, Paixão IB, Perrone AC, Monteiro MI, Santos KB. Monitorização transfusional: análise da prática assistencial em um hospital público de ensino. Einstein. 2016; 14(1):41-6. DOI: 10.1590/S1679-45082016 AO3555

4. Adamski J, Tina SI, Kinard T. Chapter 14 - Therapeutic and Donor Apheresis.Pages 2018:327-351. DOI: https://doi.org/10.1016/B978-0-12-803999-1.00014-6.

5. Brener S, Caiaffa WT, Sakurai E, Proietti FA. Fatores associados à aptidão clínica para a doação de sangue: determinantes demográficos e socioeconômicos. Rev bras hematol hemoter. 2008; 30(2):108-113

6. Costa PA, Santos P. Plasma rico em plaquetas: uma revisão sobre seu uso terapêutico. RBAC. 2016; 48.4:311-9. DOI: 10.21877/2448-3877.201600177

7. Souza WR, Spiri WC, Lima SAM, Bernardes A, Luppi CHB. Utilização do custeio baseado em atividades em centro de material e esterilização como ferramenta gerencial. Rev Eletr Enf. [Internet]. 2015 [acesso em 2019 nov 20]; abr./jun.;17(2):290-301. Disponível em: http://dx.doi.org/10.5216/ree.v17i2.27540

8. Castilho V, Fugulin FMT, Gaidzinski RR. Gerenciamento de custos nos serviços de enfermagem. In: KURCGANT, P. Gerenciamento em enfermagem. Rio de Janeiro: Guanabara Koogan; 2005. cap. 13, p. 171-183.
9. Cavassini ACM, Lima SAM, Calderon IMP, Rudge MVC. Avaliações econômicas em saúde: apuração de custos no atendimento de gestações complicadas pelo diabete. RAS. Revista de Administração em Saúde. 2010; 12(46) 23-30.

10. Branco MC et al. Caracterização de doadores de plaquetas por aférese de um hemocentro do interior de São Paulo. Revista Uningá. 2016;50:12-16.

11. Ubiali EMA et al. Custo médio do modulo de coleta de sangue pelo método $A B C$. Rev bras hematol hemoter. 2008; 30(3):213-217. DOI: http://dx.doi. org/10.1590/S1516-84842008000300010.

12. Silva Júnior JB, Rattner D, Martins RCA. Controle de riscos potenciais em serviços de hemoterapia no Brasil: uma abordagem para autoridades reguladoras. Rev Panam Salud Publica. 2016; 40(1):1-8.

13. Brasil. Ministério da Saúde. Secretaria de Atenção à Saúde. Departamento de Atenção Especializada. Segurança transfusional: um olhar sobre os serviços de hemoterapia das regiões Norte e Centro-Oeste do Brasil: III Curso de Especialização em Segurança Transfusional: resumo das monografias finais / Ministério da Saúde, Secretaria de Atenção à Saúde, Departamento de Atenção Especializada. - Brasília: Ministério da Saúde; 2012. 466 p.

14. Stein BDP et al. Avaliação da gestão da qualidade de uma agência transfusional. Revista Brasileira de Ciências da Saúde. 2017; 21.3:203-210. DOI: https:// doi.org/10.22478/ufpb.2317-6032.2017v21n3.26439 